\title{
Propagation Measurements in a Shallow Water Environment
}

\author{
Shaari M.Unger \\ Acoustic Acquisition and Analysis Division \\ Naval Undersea Warfare Center Division Keyport \\ Keyport Washington $98345-5000$
}

\author{
Raymond J. Kraszewski \\ Acoustic Acquisition and Analysis Division \\ Naval Undersea Warfare Center Division Keyport \\ Keyport Washington $98345-5000$
}

\begin{abstract}
In a 1968 study of characteristics of sound propagation in Dabob Bay, a propagation loss model for use in predicting source level was developed. With the advancement of computer technology and in the interest of improving accuracy, we have conducted a series of measurements to determine whether a more robust approximation could be used to predict source level.

A model was developed which uses input such as sound velocity, frequency, and position and is based on an iterative integration method. Transmission loss estimation methods are contrasted and compared in light of experimental losses determined from signals received by an omni-directional hydrophone.

The data acquired and the model developed illustrate the propagation characteristics of a shallow, bounded body of water and may be extended to other similar environments.
\end{abstract}

\section{INTROLIICTION}

Bolt, Beranek, and Newman (BBN) [1] demonstrated 15 $\log \mathrm{R}+\mathrm{a} \mathrm{R}$ would be an appropriate propagation loss model for use in predicting source level in 1968 . The objective of this study was to quantitatively determine whether a more robust approximation of transmission loss could be used to predict source level in Dabob Bay. The effect would be the ability to report more accurate values of source level for moving or statuonery targets at mid-bay depth.

In-situ measurements were conducted using a slowly moving point source during three distinct seasons in 1993. This paper includes a description of the environmental conditions, an explanation of the measurement methods, the effect of the velocity profile changes on the predictability of transmission loss, and details of the proposed propagation loss model.

Preliminary results of the measurements for continuous wave (cw) data at specific frequencies are presented in comparison to the model predictions. Sea surface condition was relatively smooth in all three measurements thus surface scatter is not part of this discussion.

The final criterion for acceptance of the model parameters is the agreement between propagation loss model prediction and experimental measurements for several seasonal conditions.

\section{ENVIROINMENT}

The Dabob Bay range, operated by the Naval Undersea Warfare Center Division, Keypon, is located at 47 degrees 44 minutes longitude, 122 degrees 50 minutes latitude in northwestern Washington state, U.S.A. The range site is approximately $21,0(0)$ meters by 2,500 meters with a maximum water depth of 200 meters [2]. Dabob Bay is part of Puget Sound originally formed during the glacial age out of volcanic rock. The sides of this fjord like area are steep. In the intertidal zone sand, gravel, and volcanic rock are evident. Along the hasin slope expect fine to medium sand and mud. Mud and silt are present on the bottom.

Volume reverberation is caused by both the reverberant sides of the bay and biomass within the water column. The region is shadowed by the Olympic Mountains to the west. Run-off from snow melt contributes to the summer freshwater surface layer.

The bay entrance is relatively open to the southeast. The winds are southerly during most storms and northerly during sunny weather. Streams and rivers entering Dabob Bay also reduce the salinity. Pacific Ocean seawater enters the bay through the Hood Canal.

Dabob Bay has both diumal and semidiumal components dependent upon time of month and year. The flood tide is from the south and during a tide change the water level can change as much as 5 meters in a 6 hour period. Parcels of warm or relatively fresh water remain intact for several weeks at depths of 80-100 meters during the Fall before being dissipated by flushing or intrusion of more saline water. Eventually the entire water column turns over. Then the bay once again becomes homogenous. The duration of each seasonal velocity profile is not dependent upon local weather. For example, although the region was very wet during the months of June and July 1993 followed by an unusually dry and calm August and September, the standard Summer velocity profile changed to the expected Fall profile, as shown in Figure 1.

\section{EXPERIMENT}

The objective of the experiment was to directly measure any propagation anomalies caused by multipath and sound velocity profile (SVP) structure with respect to range and frequency.

The sound source used was comprised of a Saunders Model 30 low frequency flex tensional transducer and an F56 ceramic sphere transducer. The omni-directionality of the transducers were confirmed prior to the test. This wals imperative since a directional source could cause distorted or lowered signals if the source were streaming and not vertical. The receivers were part of the Bottom Moored 


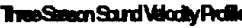

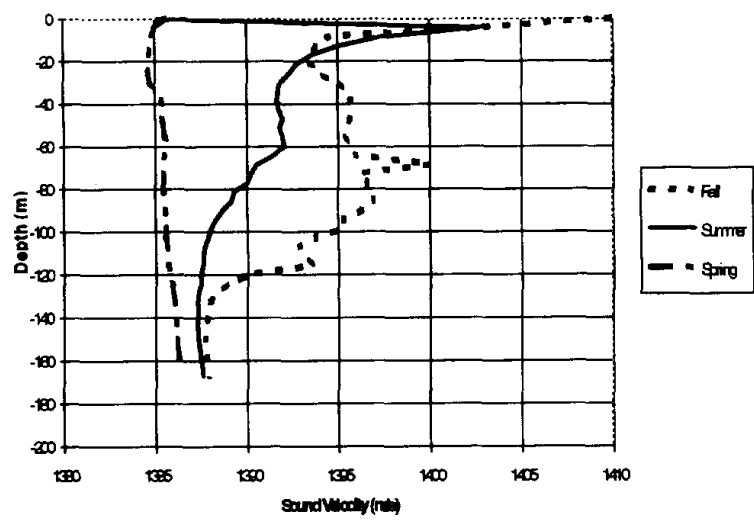

Fig. 1. Three Season Comparison in SVP

Array (BMA) which has three omni-directional hydrophones asymmetrically spaced approximately 20 and 13 meters apart vertically and almost directly above one another. The center of the line array is 2 meters below the center omni-phone. The BMA and the source were tracked with an active $3-\mathrm{D}$ pinger $(75 \mathrm{kHz})$ to determine exact location.

The source was deployed from a $21 \mathrm{~m}$ sound boat which was tied alongside a torpedo retriever (TRB) so as not to damage the source or cabling. The duo were allowed to drift. When the direction of the drift was inappropriate or the boats did not drift at all, the TRB was used to move them along at approximately 3 knots. Two types of signals were transmitted by the source. Continuous wave tones at several frequencies from $250 \mathrm{~Hz}$ to $10 \mathrm{kHz}$ were used to represent radiated noise of a moving source. Chirp data with multiple shapes and durations were used for determining the strength of the signals received after bouncing off the surface and bottom boundaries and to determine the bottom reflection coefficient.

Prior to and after each test a Neil Brown Mark III CTD conductivity, temperature and depth probe was deployed from the sound boat near the receiver to determine the SVP in the area. The sound speed in water was calculated from conductivity temperature and pressure information using the Del Grosso equation [3].

The first set of measurements was conducted in early spring when a homogenous velocity profile existed. This was considered the baseline condition. Summer measurements were conducted with a typical strong fresh water layer and homogenous body of water below the thermocline. The third set of measurements was taken in the fall with a velocity profile consisting of multiple layers from the surface to 400 feet as shown in Figure 1. The source and receiver were deployed to the same depth for each test. For the Spring and Summer tests only the 100 meter depth was used. For the Fall test several depths were chosen to investigate propagation within channels. Each drift covered 350 to 700 meters along the centerline of the range.

\section{ASSUMPTIONS AND DATA PROCESSING}

Bottom loss was expected to be relatively low based on the BBN study. The bottom reflection coefficient used was proposed by NUWC Division Newport representative Richard Menoche who was instrumental in setting up and carrying out the spring test as part of a NUWC Division Keyport/Newport joint experiment. The water surface was assumed to be lossless with a 180 degree phase shift.

Data were analyzed using the Underwater Noise Analysis Facility (UNAFAC) at NUWC Division Keyport. All data were corrected for Doppler shift and acquisition system frequency response characteristics. One third octave data were synthesized from narrowband data.

\section{PROPAGATION MOLEL}

The discussion that follows is a cursory introduction to the theoretical basis of the propagation model used in the studies conducted at Dabob Bay. For a complete exposition of the theory and results the reader may refer to [4]. The purpose of the model is to permit a quick calculation of the incoherent propagation loss in estimating the power of the source.

\section{A. An Iterative Integral Solution to the Helmholtz Equation in Inhomogeneous Media}

The primary idealization used to describe wave propagation is the wave equation,

$$
\nabla^{2} \mathrm{P}-\frac{1}{\mathrm{c}^{2}} \frac{\partial^{2} \mathrm{P}}{\partial \mathrm{t}^{2}}=0,
$$

where $c=c(x, y, z, t)$. When the media is invariant with respect to time, $c=c(x, y, z)$, a Fourier transform can he used to convert the wave equation into the Helmholtz equation,

$$
\begin{gathered}
\quad \nabla^{2} P=\kappa P, \\
\text { where } \kappa=(\omega / c(x, y, z))^{2} .
\end{gathered}
$$

One general solution to the three-dimensional wave equation in inhomogeneous media, may be expressed as a series of functions.

$$
P=1+\sum P_{n}: \nabla^{2} P_{n}=(-1)^{n} \kappa P_{n-1}:
$$




$$
\nabla^{2} P_{0}=\kappa
$$

The first term in this summation is a solution to Poisson's equation with the wave number corresponding to a source. Subsequent terms are generated by solving Poisson's equation iteratively. The product of each term in the summation times the wave number serves as the source which generates the next term. The series solution generated in this way converges quickly however, in most cases, its implementation requires numerical integration.

\section{B. Horizontally Stratified Media}

As an illustration, when propagation occurs in a media in which the propagation variable is a function of one spatial variable, $\mathrm{z}$, which may correspond to depth, two independent plane wave solutions to the Helmholtz equation can then be expressed as a series of iterated integrals.

$$
\begin{aligned}
P_{1} & =C_{1}\left(1-\int j \kappa+\iint \kappa \int j \kappa-\cdots\right) \\
P_{2} & =C_{2}\left(z-\iint z \kappa+\iint \kappa \iint z \kappa-\cdots\right)
\end{aligned}
$$

When the wave number is constant these series converge to the trigonometric functions, $\cos$ and sin, respectively.

In many situations the wave number, which is a function of the sound velocity profile can be expressed as a finite Taylor series, $\kappa=\kappa_{0}+\kappa_{1} z+\kappa_{2} z^{2}+\cdots \kappa_{n} z^{n}$, with a high degree of accuracy. Applying this series to equations 4 and 5 , the solutions can be expressed in the following manner.

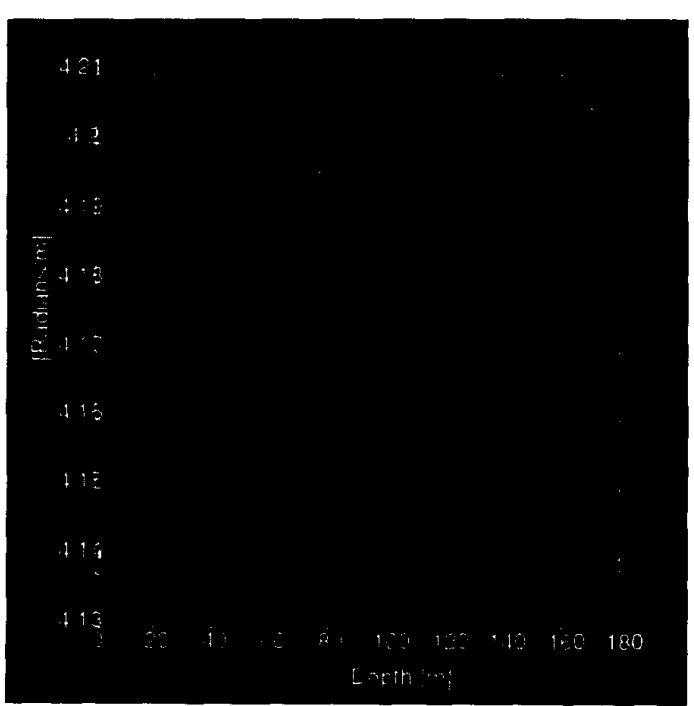

Fig.2. Wave Number From July 1993 SVP Measurements

$$
\begin{aligned}
& P^{(1)}(z)=\sum P_{n}^{(1)} \quad: P_{n}^{(1)}=z^{2 n-1} \sum a_{n, i} z^{n} \\
& a_{i, n}=\frac{(n+2 i-3) !}{(n+2 i-1) !} \sum_{m=1}^{n} \kappa_{m} a_{i-1, n-m+1} \\
& P^{(2)}(z)=\sum P_{n}^{(2)} \quad: \quad P_{n}^{(2)}=z^{2 n} \sum b_{n, i} z^{n} \\
& b_{i, n}=\frac{(n+2 i-2) !}{(n+2 i) !} \sum_{m=1}^{n} \kappa_{m} b_{i-1, n-m+1}
\end{aligned}
$$

\section{IMPL.EMENTA TION}

The sound velocity profile was measured at Dabob Bay in July of 1993. At the same time the sound fields of signals radiating with a field strength of 140 to $150 \mathrm{~dB}$ ref $\mu \mathrm{P}$ at $.25-10 \mathrm{kHz}$ were probed at various positions on range. The SVP measurements were taken at multiple depths, assuming that the media was horizontally stratified so that the fields were modeled using iterated integrals. The propagation variable, as shown in Figure 2, was modeled by a fourth degree polynomial. Boundary conditions were resolved using images above and below the water surface and the sedimentary bottom. Figure 3 depicts the sound measurements acquired at the receiving hydrophone.

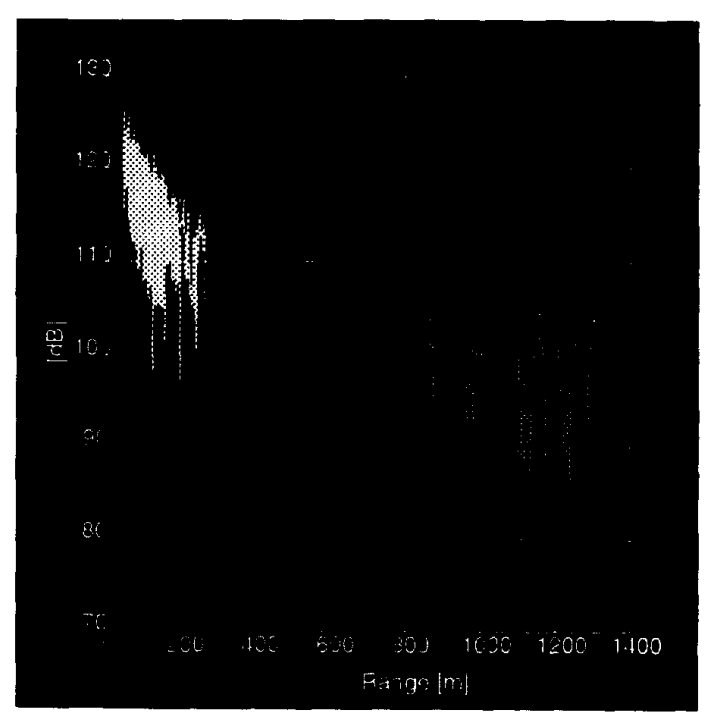

Fig.3. Received Levels for $1 \mathrm{kHz}$, July 1993 Experiments 


\section{SUMMARY AND CONCLIUSIONS}

The larger the sample size from which averages of spectral estimates of a moving source are derived, the more accurately the BBN propagation loss model becomes. This is to be understood in the sense of convergence. Samples taken over a long period of time and with multiple receivers can be used to resolve the variability of the multipath signals. Differences between the Spring, Summer, and Fall results are due to the SVP. The Fall multilayer SVP causes sound ducting. Significant fluctuations due to the Lloyd Mirror effect cause multipath signals alternately to amplify and nullify. This is illustrated in Figure 3 where the variation in sound levels at the receiver is attributed to spatial variations in the radiating sound field despite the fact that the source level is fixed. By averaging the incoming signal of a set of omni-phones over a long time span this effect can be minimized.

The practical problem is that with a moving source it is not always possible to take averages over a long period of time and multiple receivers are not always available. The proposed model may be used with one hydrophone when environmental parameters are well understood. The model assumes three things. One, sound velocity is accurately measured, two, the positions of the source and the receiver are accurately known and three, the boundary conditions are well defined. Given these parameters it is believed that the accuracy of the proposed model can be as high as $1 \mathrm{~dB}$ when applied to the dynamic noise measurements conducted in Dabob Bay.

These are the preliminary conclusions for the omniphone data. The chirp data collected will be processed to determine the bottom reflection coefficient as well as the loss contributed by the boundaries. Further measurements can be made using this same process in this same location as well as other shallow water areas to validate our conclusions and form a data base.

In further studies the question of surface roughness will have to be addressed with respect to season. In summer the mixed surface layer and thermocline decreases the chance of refraction from surface interaction and increases the bottom interaction. In Spring there is no high speed layer to shield the surface, so losses due to surface scattering will be more pronounced especially at high frequency. Future tests would include depth variation and multiple SVP's.

We require a better understanding of sea surface, water column, and sea-floor parameters measured together with acoustic propagation to obtain a more thorough understanding of shallow-water acoustic propagation.

\section{ACKNOWLEDGMENTS}

This research was performed under the sponsorship of the United States Naval Sea Systems Command.

\section{REFERENCES}

[1] Bolt, Beranek and Newman "Prop loss modeling of the Scotian Shelf, comparison of model predictions with measurements"

[2] R. Helton "Oceanographic and acoustic characteristics of the Dabob Bay range" NavTorpSta Report 1300 Naval Torpedo Station, Keyport, WA (1976)

[3] V.A. Del Grosso, "New equation for the speed of sound in natural waters ( with comparisons to other equations), " J. Acoust.Soc.Am., Vol.56, No. 4, October 1974

[4] R.Kraszewski, "An iterative method for the solution of the Helmholtz equation in stationary inhomogeneous media," unpublished. 